\title{
Bionic Design of Wind Turbine Blade Based on Long-Eared Owl's Airfoil
}

\author{
Weijun Tian, ${ }^{1,2}$ Zhen Yang, ${ }^{1}$ Qi Zhang, Jiyue Wang, ${ }^{1}$ Ming Li, ${ }^{1}$ Yi Ma, ${ }^{3}$ and Qian Cong1,2 \\ ${ }^{1}$ Key Laboratory of Bionic Engineering (Ministry of Education, China), Jilin University, Changchun 130022, China \\ ${ }^{2}$ State Key Laboratory of Automotive Simulation and Control, Jilin University, Changchun 130022, China \\ ${ }^{3}$ China Automotive Engineering Research Institute Co., Ltd., Chongqing 401122, China \\ Correspondence should be addressed to Qian Cong; congqian@jlu.edu.cn
}

Received 8 September 2016; Accepted 10 November 2016; Published 22 January 2017

Academic Editor: Luis Gracia

Copyright (C) 2017 Weijun Tian et al. This is an open access article distributed under the Creative Commons Attribution License, which permits unrestricted use, distribution, and reproduction in any medium, provided the original work is properly cited.

\begin{abstract}
The main purpose of this paper is to demonstrate a bionic design for the airfoil of wind turbines inspired by the morphology of Long-eared Owl's wings. Glauert Model was adopted to design the standard blade and the bionic blade, respectively. Numerical analysis method was utilized to study the aerodynamic characteristics of the airfoils as well as the blades. Results show that the bionic airfoil inspired by the airfoil at the $50 \%$ aspect ratio of the Long-eared Owl's wing gives rise to a superior lift coefficient and stalling performance and thus can be beneficial to improving the performance of the wind turbine blade. Also, the efficiency of the bionic blade in wind turbine blades tests increases by $12 \%$ or above (up to $44 \%$ ) compared to that of the standard blade. The reason lies in the bigger pressure difference between the upper and lower surface which can provide stronger lift.
\end{abstract}

\section{Introduction}

Wind power is a clean and renewable source of energy [1]. The increasing use of wind power is of great significance for reducing environmental pollution, improving energy consumption, and thus realizing sustainable development. A key component of wind turbines is blades, which consist of different airfoil surfaces. The aerodynamic characteristics of airfoils have direct influence on the performance of the blades, and thus the airfoils are crucial to blade design. Early traditional wind turbine blades were based on aeronautic applications, for example, NACA series, but later airfoils specifically designed for wind turbines were developed by NREL, Risø, FFA, and so forth [2-5]. The specialized airfoil series greatly changed the operating conditions of wind turbines and increased the blade adaptability. This led to a startup at low wind speeds as well as a stall delay at high wind speeds. Most of these airfoils were developed using traditional inverse design approaches [6]. In practice, the function of birds' wings is very similar to that of wind turbine blades [79]. The bionic research finds that the airfoils inspired by birds, including the seagull airfoil and those based on the leading edge of Long-eared Owl wings, have better aerodynamic performance [10-13].

In this work, a new airfoil of the wind turbine blades was designed and used in the wind turbine blades. The efficiency of the wind turbine blades was carried out, and it was investigated how the new airfoil influences the performance of blades by aerodynamics numerical simulation.

\section{Materials and Methods}

2.1. Bionic Airfoil Extraction. The point cloud data of the airfoils were collected using a 3D scanning system (Rexcan III, Solutionix Corp., Korea) with a scanning accuracy of $0.001 \mathrm{~mm}$. The wing of the Long-eared Owl was unfolded naturally before the experiment. The scanned data were processed in IMAGEWARE 12, and the airfoil with superior aerodynamic performance was named as "bionic airfoil," which is located at the point with a span-wise aspect ratio of $50 \%$ on the wings [11]. The airfoil curve obtained after fairing is shown in Figure 1. 


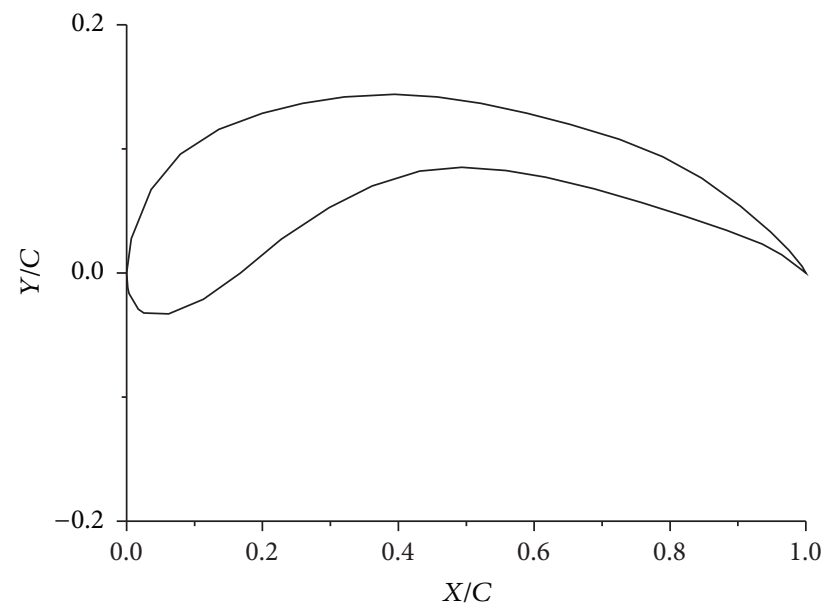

FIGURE 1: Curve of the bionic airfoil.

TABLE 1: Parameters of the bionic airfoil.

\begin{tabular}{lc}
\hline Variable & Value \\
\hline Chord length & $c$ \\
Maximum thickness as a fraction of the chord $(t)$ & $0.1306 c$ \\
Leading edge radius $(r)$ & $0.047355 c$ \\
Camber $(f)$ & $0.1127 c$ \\
Position of the maximum thickness $\left(x_{t}\right)$ & $11.3 \% c$ \\
Position of the maximum camber $\left(x_{f}\right)$ & $43.1 \% c$ \\
\hline
\end{tabular}

The profile geometry of the bionic airfoil is shown in Figure 2, the chord length is $c$, and the geometry parameters are shown in Table 1.

\subsection{Blade Design}

2.2.1. Standard Blade Reconstruction. NACA4412 is one of the most common airfoils used for wind turbine blades due to its superior aerodynamic characteristics. A 500 Watt wind turbine blade of the NACA4412 airfoil was selected as "standard blade." The length of the blade is $1200 \mathrm{~mm}$, the blade root is from $0 \mathrm{~mm}$ to $130 \mathrm{~mm}$ and the blade span is from $130 \mathrm{~mm}$ to $1200 \mathrm{~mm}$ along the $x$-axis. In particular, the blade span of the standard blade is divided into 12 equal sections (see Figure 3 ), and the height of the section $A-A$ is $\Delta h$. The airfoil shape of every section was obtained using the scanner, and the angle of attack (AOA) $\alpha=\arcsin (\Delta h / c)$ was calculated after measuring the chord length $c$.

Airfoil at each section was established using CATIA V5R19. The aerodynamic center lies exactly at the one-quarter position of the chord. The blade was constructed by rotating the airfoils around the aerodynamic center according to the AOA, as shown in Figure 4.

2.2.2. Bionic Blade Design. Because the characteristics of the airfoils used over the tip region of the blades have an important impact on the efficiency of the wind turbine, the airfoils of the chord positions after $60 \%$ were designed using
TABLE 2: Design parameters of the bionic blade.

\begin{tabular}{lccc}
\hline Rated power $(P)$ & $\begin{array}{c}\text { Diameter } \\
(D)\end{array}$ & $\begin{array}{c}\text { Number of } \\
\text { blades }(B)\end{array}$ & $\begin{array}{c}\text { Tip-speed ratio } \\
\left(\lambda_{0}\right)\end{array}$ \\
\hline $500 \mathrm{w}$ & $1.2 \mathrm{~m}$ & 3 & 6 \\
\hline
\end{tabular}

the bionic airfoils. The design parameters of the bionic blade are shown in Table 2.

The lift-to-drag ratio of the airfoil reached its maximum at the span-wise aspect ratio of $50 \%$, when the AOA was set at $3^{\circ}$ [11], which in turn was used for the blade design. The blade incidences $(\theta)$ of different span-wise positions were calculated according to the Glauert theory [14, 15], and the bionic blade and standard blade should have the same chord length to make sure that they have the same solidity. The constructed model of the bionic blade was shown in Figure 5.

\subsection{Aerodynamics Numerical Simulation}

2.3.1. Numerical Simulations on Aerodynamics of the Airfoils. In ANSYS14.5, CEM-CFD was used to divide grid and FLUENT was used to carry out the numerical simulations for the standard airfoil (NACA4412 airfoil) and the bionic airfoils. Reynolds number is defined as follows:

$$
\operatorname{Re}=\frac{\rho v c}{\mu},
$$

where Re is Reynolds number, $v$ is the mean velocity of the object relative to the fluid $(27 \mathrm{~m} / \mathrm{s}), c$ is the chord length $\left(3 \times 10^{-2} \mathrm{~m}\right.$ to $\left.4.5 \times 10^{-2} \mathrm{~m}\right), \rho$ is the density of the fluid $\left(1.225 \mathrm{~kg} / \mathrm{m}^{3}\right)$, and $\mu$ is the dynamic viscosity of the fluid $\left(1.789 \times 10^{-5} \mathrm{~kg} / \mathrm{m} \cdot \mathrm{s}\right)$. According to the (1), the range of Reynolds number is between $5.55 \times 10^{4}$ and $8.32 \times 10^{4}$. In our numerical simulation, Reynolds numbers $(\mathrm{Re})$ were $6 \times 10^{4}$ and $8 \times 10^{4}$.

The large eddy simulation method was adopted for the numerical simulations. The computed field is shown in Figure 6. The airfoil chord length is $C$. The left field is a semicircle with a radius of $12.5 C$, and the right part is a rectangle with a height of $20 C$ and a width of $25 C$. When Mach Number (Ma) is less than 0.3, the air is considered as incompressible flow. Therefore, the inlet and outlet were set to "velocity inlet" and "pressure outlet," respectively. The meshes generated were $C$-type meshes, as shown in Figure 7 (a). The local mesh refinement was implemented at the leading edge and trailing edge of the airfoil, as shown in Figure 7(b).

Spalart-Allmaras model (S-A) is a simple-equation turbulence model which solves the turbulence viscosity of the transport equations. Boundary layers that cover the airfoil are divided into the viscous sublayer, the buffer layer, and the logarithm sublayer. The velocity gradient and viscous force of the viscous sublayer are large. As a result, in order to obtain the accurate solutions of the viscous sublayer, the grid must be sufficiently fine, which means the wall coordinate $y^{+}$is less than 5. In our simulation, the thickness of the first layer was approximately 0.0002 , and $y^{+}$was less than 1 . 


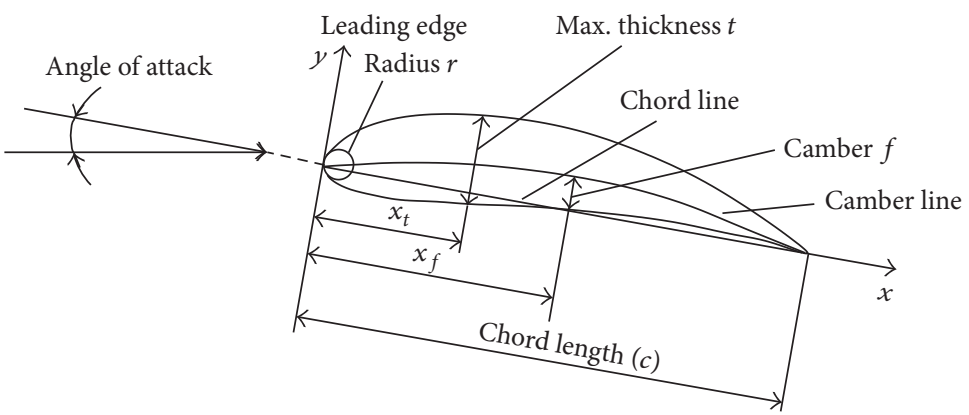

Figure 2: Profile geometry of the bionic airfoil.

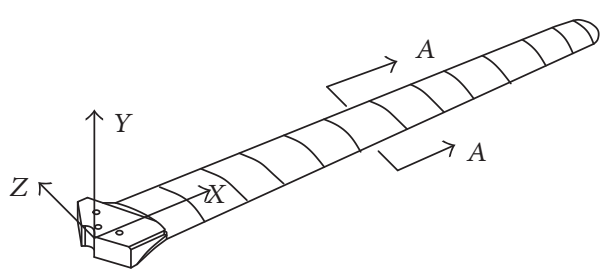

(a)

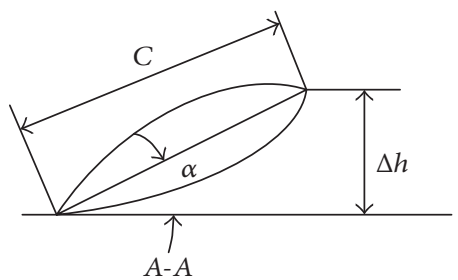

(b)

Figure 3: Divisional schematic (a) of a wind turbine blade and the $A$ - $A$ cross-sectional profile (b).

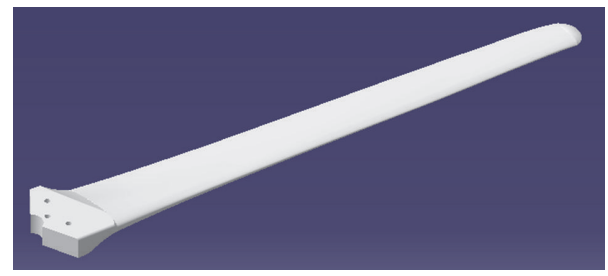

FIGURE 4: Model of a standard blade.

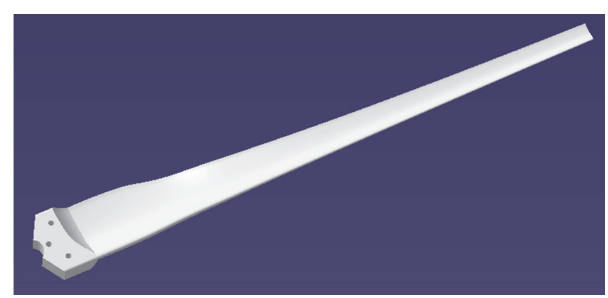

FIGURE 5: Model of bionic blade.

Figures 8 and 9 summarize the results for the mesh sensitivity of the computational grid study. Here the results are presented for the lift coefficients $\left(C_{l}\right)$ and drag coefficients $\left(C_{d}\right)$ of $\mathrm{Re}=6 \times 10^{4}$ and $8 \times 10^{4}$ using three grid sizes of 22425 , 33400 , and 63000 nodes. Based on this grid dependence study, it is concluded that the medium grid size of 33400 is sufficient for carrying out the simulations.

2.3.2. Numerical Simulations on Aerodynamics of the Blades. The simulation of the wind turbine blades was conducted, and the model was simplified to reduce the calculation complications. The influences of the tower and engine-room

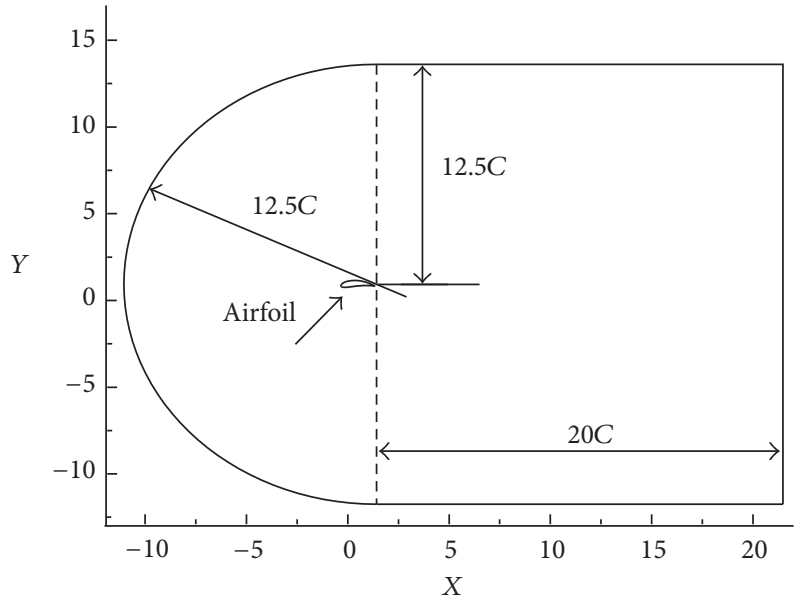

FIGURE 6: Computational domain.

were ignored. The diameter of the cylindrical domain used for simulation was $2 R$, twice as large as the radius of the blade. The domains of the inlet and outlet were $R$ and $4 R$, respectively. Stationary and rotating regions were adopted as the computed domains, and the rotating region was adopted the dynamic grids. The main boundary conditions including inlet, outlet, and wall were defined. The inlet was set to "velocity inlet" and outlet was set to "pressure outlet." The velocity of inlet was set to $9 \mathrm{~m} / \mathrm{s}$, and the rotation speed of blades was set to $350 \mathrm{rpm}$. The pressure of outlet was atmosphere pressure without any additional pressure. The relative pressure of the outlet was $0 \mathrm{~Pa}$. The SST $k-\omega$ model was used in flow field analysis. The wall condition was set to smooth wall with no slip and no penetration. The simulation 


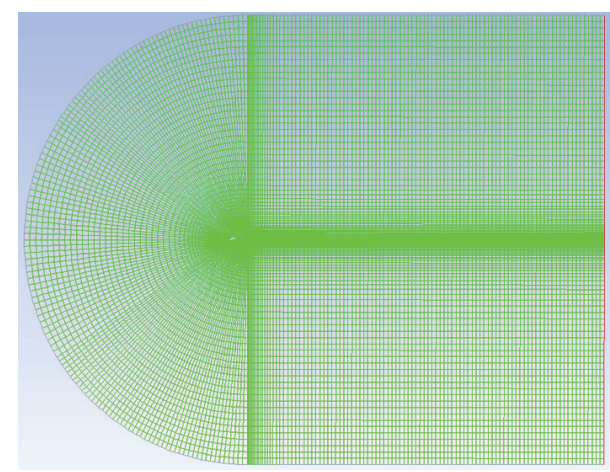

(a) Computational grid of airfoil

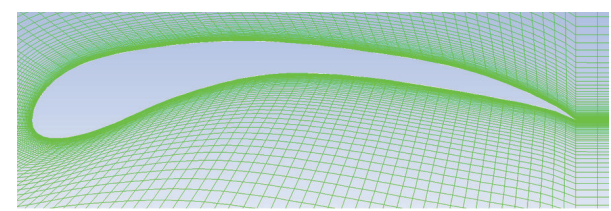

(b) Detail of the computational grid

FIGURE 7: Computational grid.

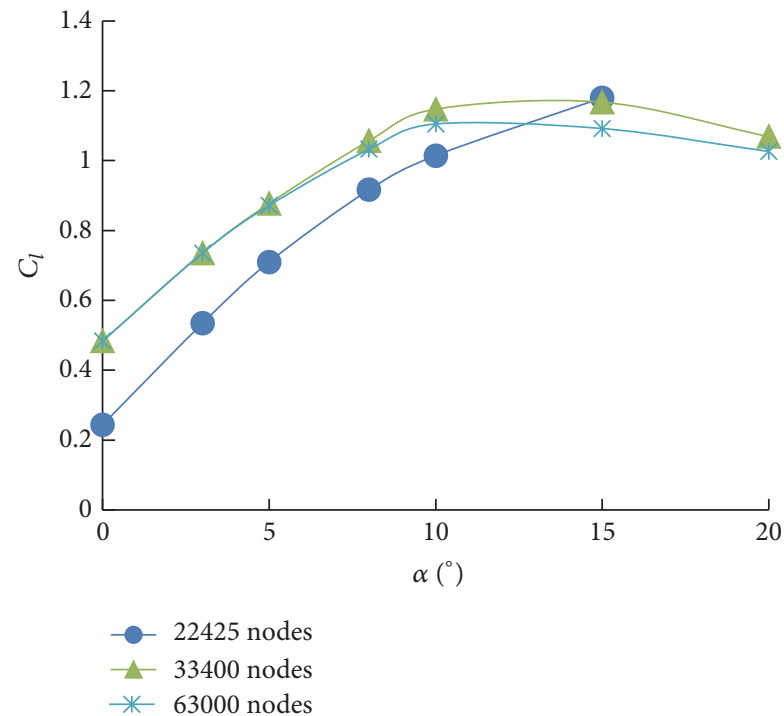

(a)

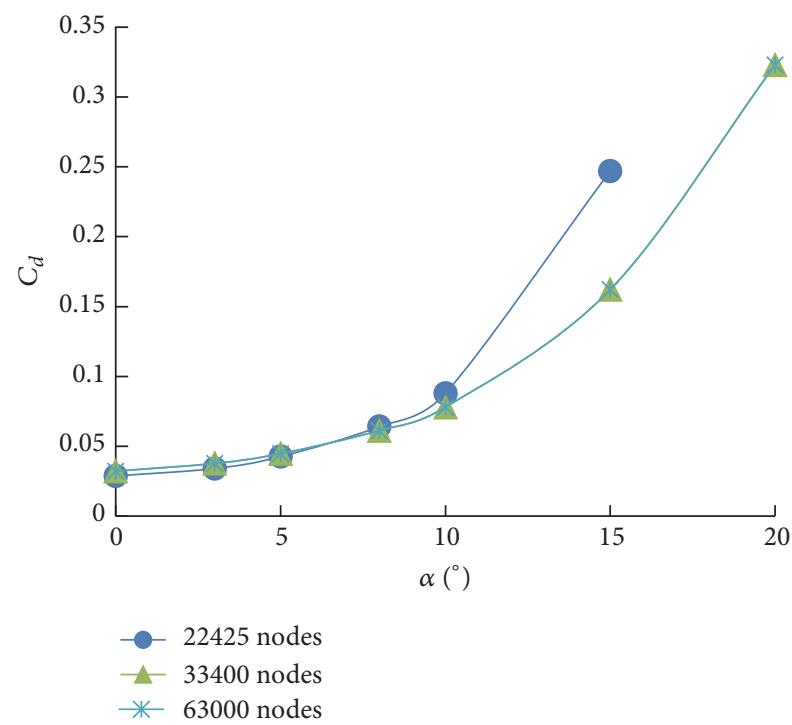

(b)

FiguRE 8: Comparison of simulation results of three grid resolutions of coarse (22425 nodes), medium (33400 nodes), and fine (63000 nodes) meshes at $\operatorname{Re}=6 \times 10^{4}$. (a) Lift coefficients $\left(C_{l}\right)$ and (b) drag coefficients $\left(C_{d}\right)$.

domains and boundary conditions are shown in Figure 10. The calculation adopted unstructured grids and the quantity of the blade grids was $2.3 \times 10^{6}$.

2.4. Efficiency Experiment of Blades. Wood was selected as the blade materials, and wood engraving machine XJ-1218 was selected to machine the blades. 3D blades were generated via CATIA and G codes were imported into the engraving software for machining the blades. The blade surfaces were polished after being machined.

The efficiency experiments of the blades were carried out in natural wind. The test system was composed of wind turbine, rectifier stack, anemometer, electronic load, oscilloscope, and PC, as seen in Figure 11. The electronic load was used to test the voltage, electric current, and power. Anemometer was used for the testing of the real-time wind speeds. Oscilloscope was used as frequency meter in system to collect the Alternating Current pulse frequency and then converted to rotational speed of the wind turbine blade. The parameters such as velocity, voltage, and electric current of the wind turbine were recorded at different wind speeds in the range of $2-10 \mathrm{~m} / \mathrm{s}$, and the wind power coefficient $C_{p}$ was calculated based on the wind power formula.

\section{Results}

The power curves shown in Figure 12 describe the relationship between wind speed and power output. It shows that there is not enough torque exerted by the wind on the turbine blades to make them rotate at very low wind speeds (below $3 \mathrm{~m} / \mathrm{s}$ ). As the wind speed rises above the cut-in speed $3 \mathrm{~m} / \mathrm{s}$, the power rises rapidly. The power of the bionic wind turbine (the wind turbine with bionic blades) is larger than that of the standard wind turbine at the same wind speed. When the speed reaches $7 \mathrm{~m} / \mathrm{s}$, the power of the bionic wind turbine is $320 \mathrm{~W}$ while the standard wind turbine is $265 \mathrm{~W}$. The results 


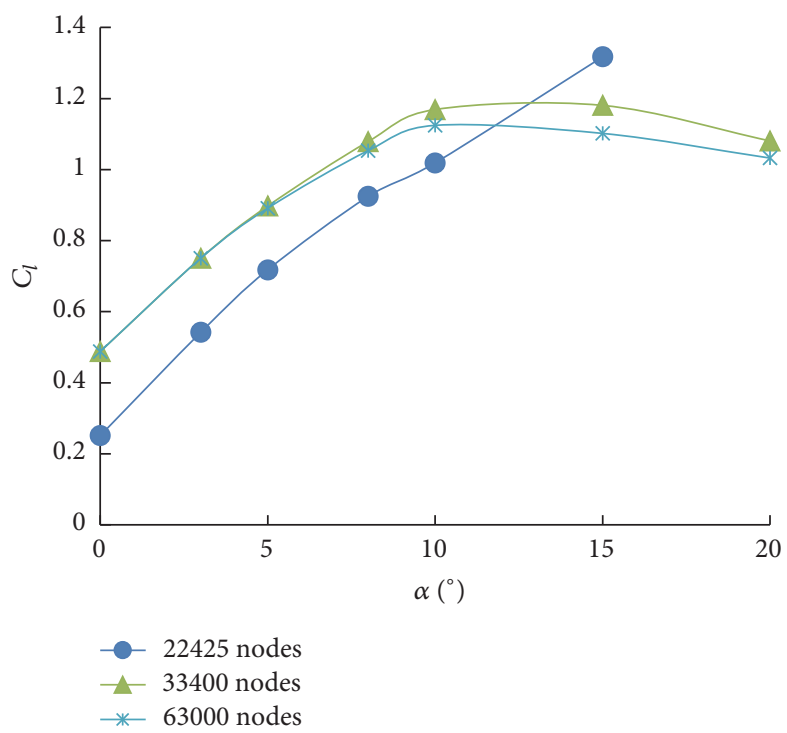

(a)

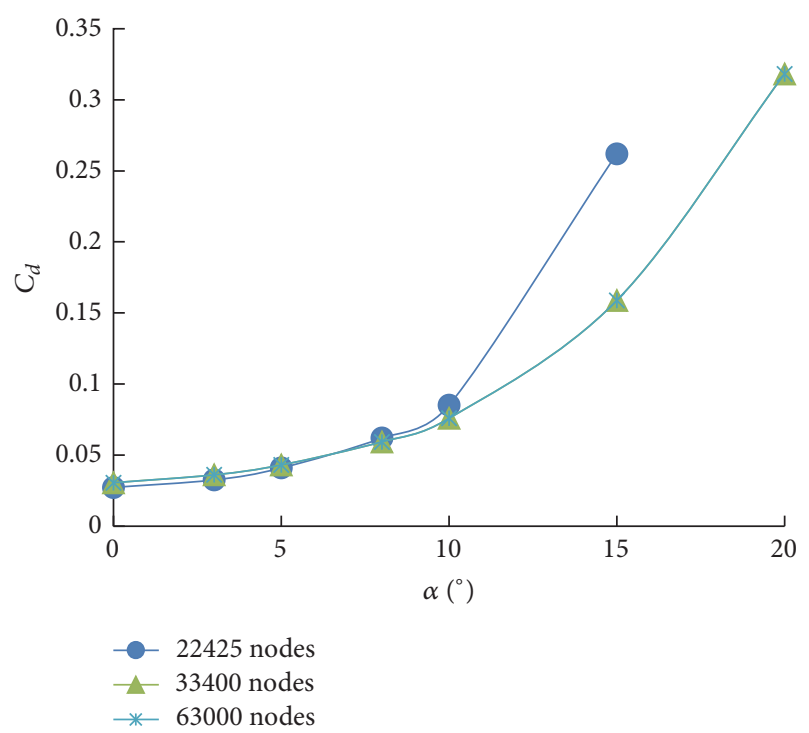

(b)

FIGURE 9: Comparison of simulation results of three grid resolutions of coarse (22425 nodes), medium (33400 nodes), and fine (63000 nodes) meshes at $\operatorname{Re}=8 \times 10^{4}$. (a) Lift coefficients $\left(C_{l}\right)$ and (b) drag coefficients $\left(C_{d}\right)$.

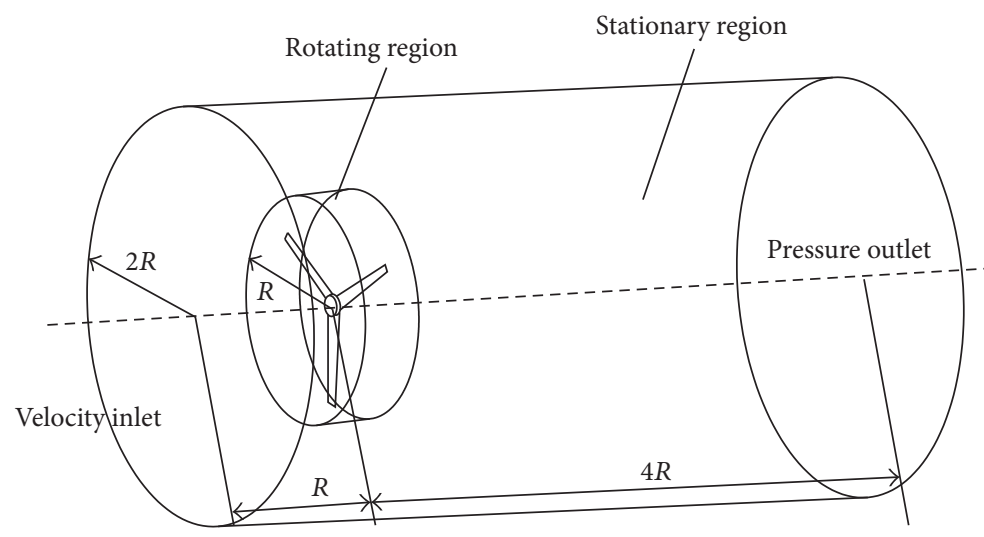

FIGURE 10: Simulation domains and boundary conditions.

indicate that the bionic blade increases the efficiency of the wind turbine and utilizes the wind power more efficiently.

The power coefficient $C_{p}$ of the wind turbines at different wind speeds is show in Figure 13. As the wind speed rises, the $C_{p}$ increases to a maximum of 0.35 for the standard wind turbine and 0.44 for the bionic wind turbine and then decreases. The results indicate that the bionic wind turbine has the superior aerodynamic characteristics at the lower wind speeds.

\section{Discussion}

Bionic wind turbine has superior characteristics, so it is necessary to study its mechanism. Because the blade airfoil is one of the decisive factors in wind turbine performance, it is necessary to analyze and compare the airfoils' aerodynamic characteristics of the standard blades and the bionic blades to find the reason that leads to the performance improvement of the bionic blades.

Comparison of the mean cambers of the standard airfoil and the bionic airfoil is shown in Figure 14. The relative camber $\bar{f}_{1}$ of bionic airfoil is larger than that of standard airfoil. The larger camber makes the velocity difference increase, and it results in larger pressure difference to raise the lift.

The lift coefficients $\left(C_{l}\right)$ and drag coefficients $\left(C_{d}\right)$ generated of standard airfoil and the bionic airfoil by numerical simulations at the different Reynolds numbers $(\operatorname{Re}=6 \times$ $10^{4}, \operatorname{Re}=8 \times 10^{4}$ ) are shown in Figure 15. The $C_{d}$ and $C_{l}$ of two Reynolds numbers have little difference, apart from the fact that the $C_{l}$ of the higher Reynolds number is higher while the $C_{d}$ of it is lower. However, the bionic airfoil has the same stability as standard airfoil. As the AOA, $\alpha$, increased, within the range of $0^{\circ}-10^{\circ}$, the $C_{l}$ and $C_{d}$ gradually increased. 


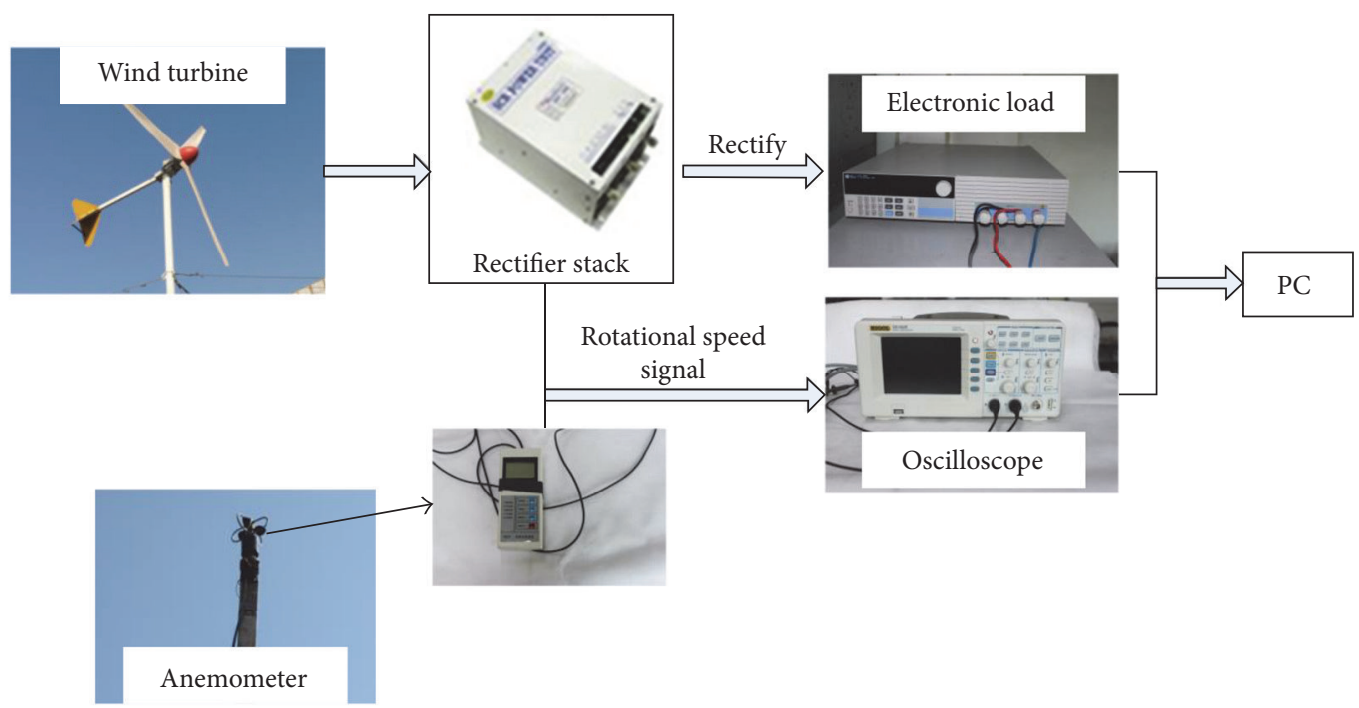

Figure 11: Test system.

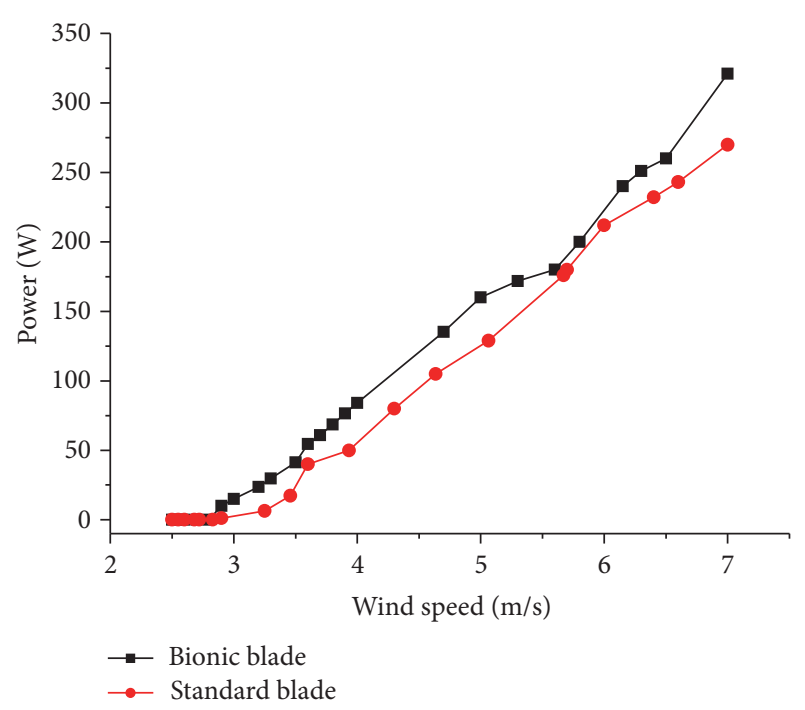

FIGURE 12: Relationships between power and wind speeds.

The maximum value of the $C_{l}$ was obtained at $\alpha=10^{\circ}$, and then, as $\alpha$ increased, the $C_{l}$ gradually decreased while the $C_{d}$ increased substantially, which means the airfoil is in the stall condition at AOA of $10^{\circ}$.

Due to the viscous resistance at the boundary layer of the airfoil surface, as well as the airflow that continuously enters in from the outside, the airflow cannot move on the airfoil surface. Instead, it separates and gradually generates air pressure with the AOA increasing. This causes the backflow of air. Subsequently eddy current is formed on the airfoil surface and the stall is generated. The airflow loses the airfoil effect and decreases the pressure difference between the upper and lower surfaces, thus resulting in the soaring of the $C_{d}$ and decreasing of the $C_{l}$.

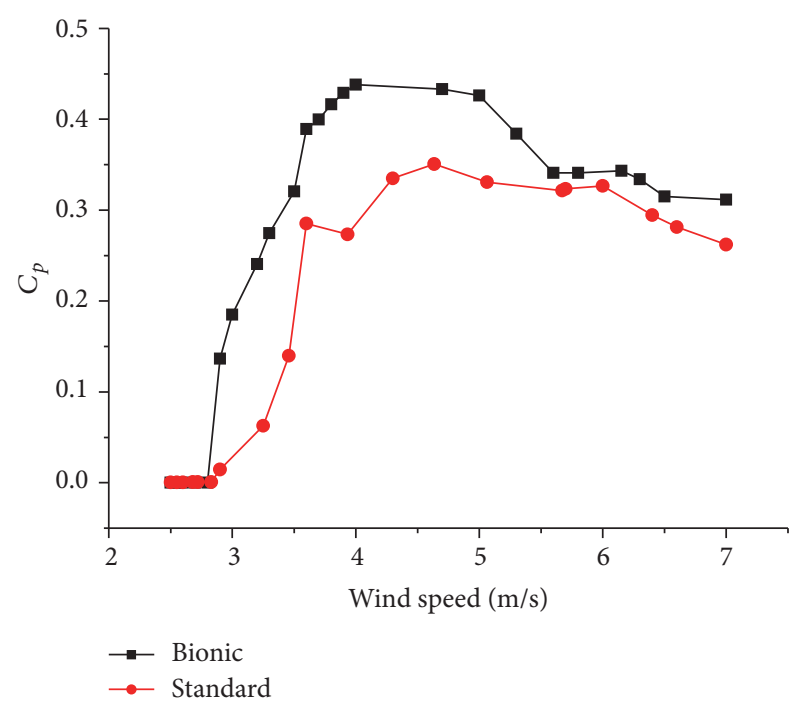

FIgURE 13: $C_{p}$ of the wind turbines at different wind speeds.
Compared with the standard airfoil, the bionic airfoil showed a higher and thus better $C_{l}$. The reason was the increase in the effective AOA of the bionic airfoil, due to the effective bending increase, as well as in the pressure difference between the upper and lower surfaces.

Figure 16 shows the lift-to-drag ratio curves of the bionic airfoil and the standard airfoil. It is noticed that their trends were basically consistent. The main difference, however, was that the curves of the bionic airfoil moved forward and reached the maximum value prior to the standard airfoil (the AOA of the maximum lift-to-drag ratio was smaller). The maximum values were 19.3 for the bionic airfoil and 19.6 for the standard airfoil, at $\mathrm{Re}=6 \times 10^{4}$, and 20.8 and 20.9 at $\mathrm{Re}=$ $8 \times 10^{4}$, respectively. 


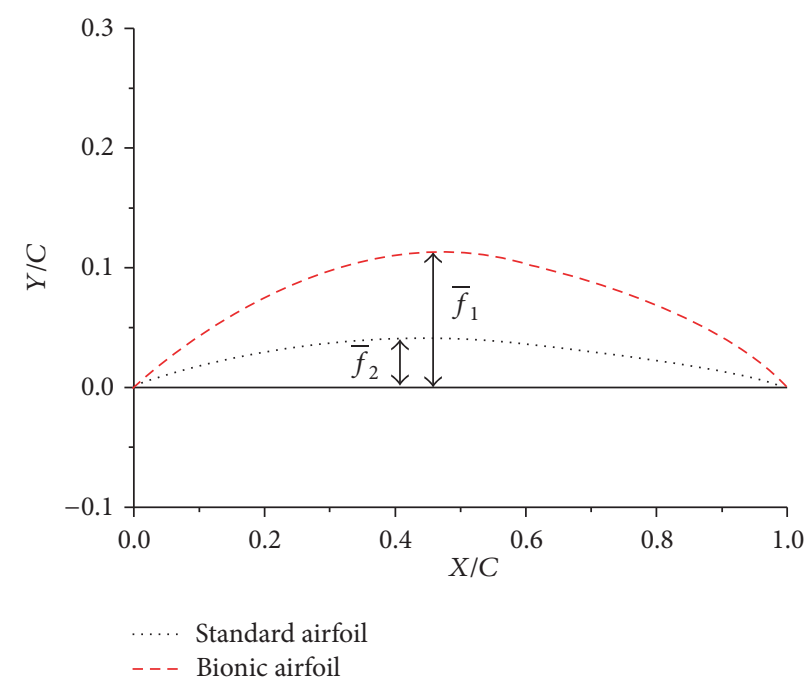

FIgURE 14: Mean cambers of the airfoils.

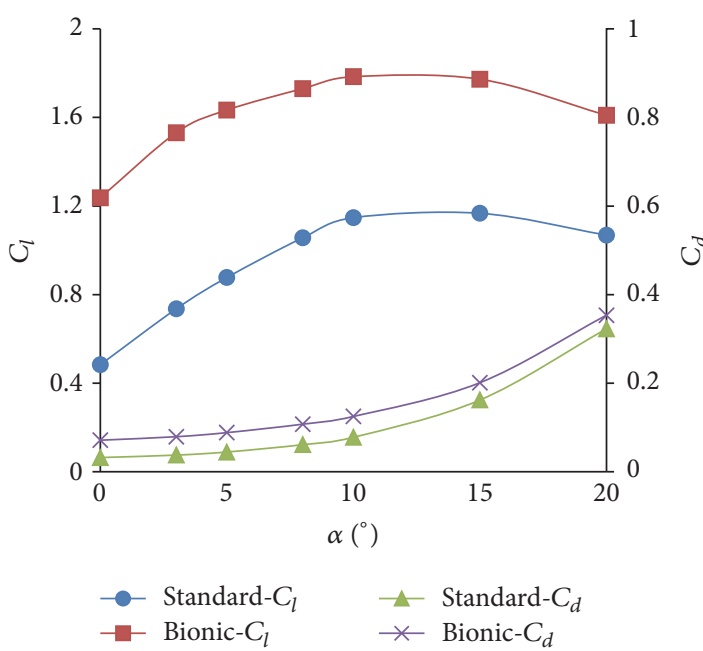

(a) $\operatorname{Re}=6 \times 10^{4}$

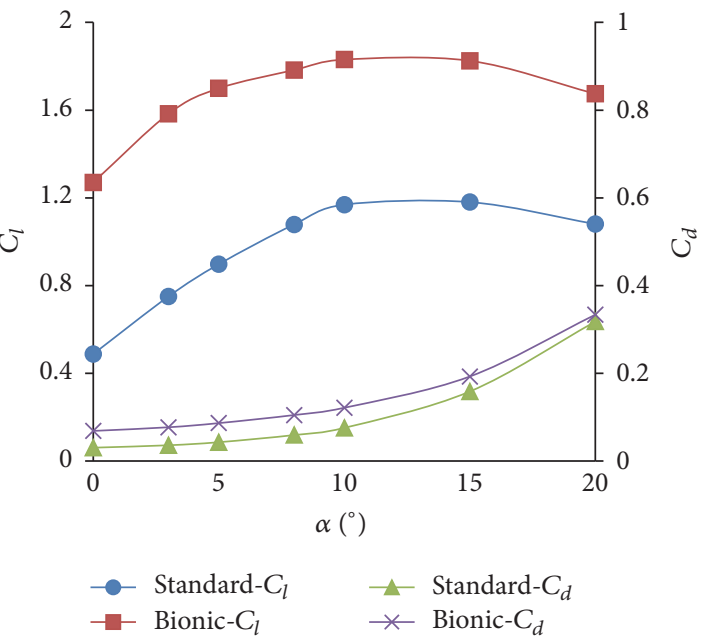

(b) $\operatorname{Re}=8 \times 10^{4}$

Figure 15: Lift coefficients $\left(C_{l}\right)$ and drag coefficients $\left(C_{d}\right)$ of the airfoils at different AOA. "Standard- $C_{l}$ " is the lift coefficient of the standard airfoil, and "Standard- $C_{d}$ " is the drag coefficient of the standard airfoil. "Bionic- $C_{l}$ " is the lift coefficient of the bionic airfoil, and "Bionic- $C_{d}$ " is the drag coefficient of the bionic airfoil.

Since the stall AOA is $10^{\circ}$, the velocity vectors colored by velocity magnitudes at the large $\mathrm{AOA}\left(10^{\circ}, 15^{\circ}\right.$ and $\left.20^{\circ}\right)$ were checked to investigate what would happen after the stall. As shown in Figures 17 and 18, for both Reynolds numbers of $6 \times 10^{4}$ and $8 \times 10^{4}$, the range of separation region of the standard airfoil was larger than that of the bionic airfoil. In addition, the range of the stall of the upper surface reached to the leading edge at $20^{\circ}$. That means the stall characteristics of the bionic airfoil are better than that of the standard airfoil. This is due to the larger camber of the trailing edge on the bionic airfoil that leads to the airflow deflecting downward. The deflection increases the momentum of the airflow at the trailing edge of the airfoil and allows the airflow to easily overcome the adverse pressure gradient at the trailing edge of the upper surface which decreases the separation zone of the upper surface.

From the comprehensive comparison of the $C_{l}, C_{d}$, liftto-drag ratio curves, and stall characteristics of the bionic airfoil and the standard airfoil, respectively, it can be noticed that the bionic airfoil has better aerodynamic performance than the standard airfoil. This, in turn, is beneficial for the improvement of the bionic blade.

The static pressures on the windward surfaces of the standard blade and the bionic blade under the wind speed of $9 \mathrm{~m} / \mathrm{s}$ are shown in Figure 19 (those airfoils are $0.9 \mathrm{~m}$ away from the root of the blades). The pressure difference of the airfoil between the upper and lower surfaces at the tip of the bionic blade is obviously higher than that of the 


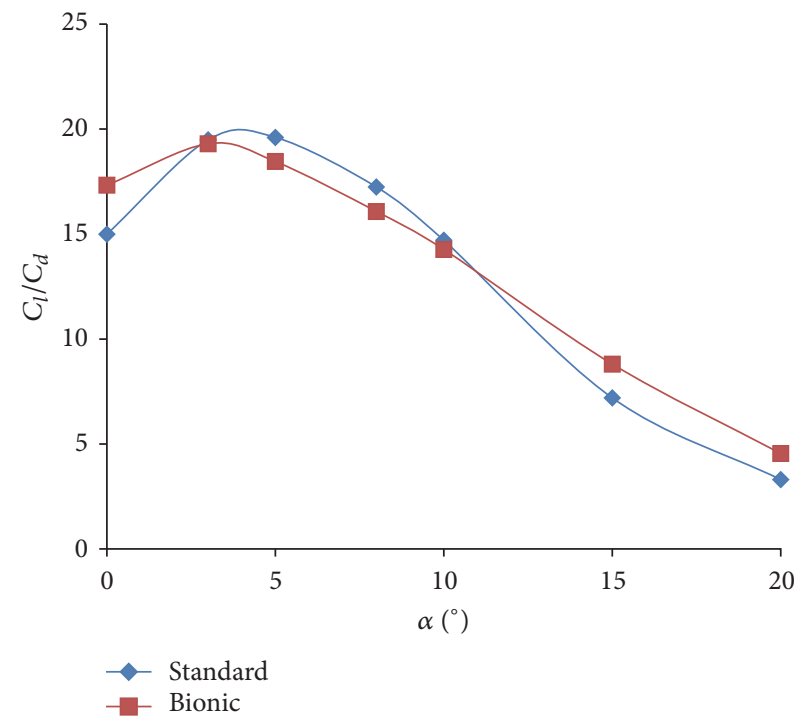

(a) $\operatorname{Re}=6 \times 10^{4}$

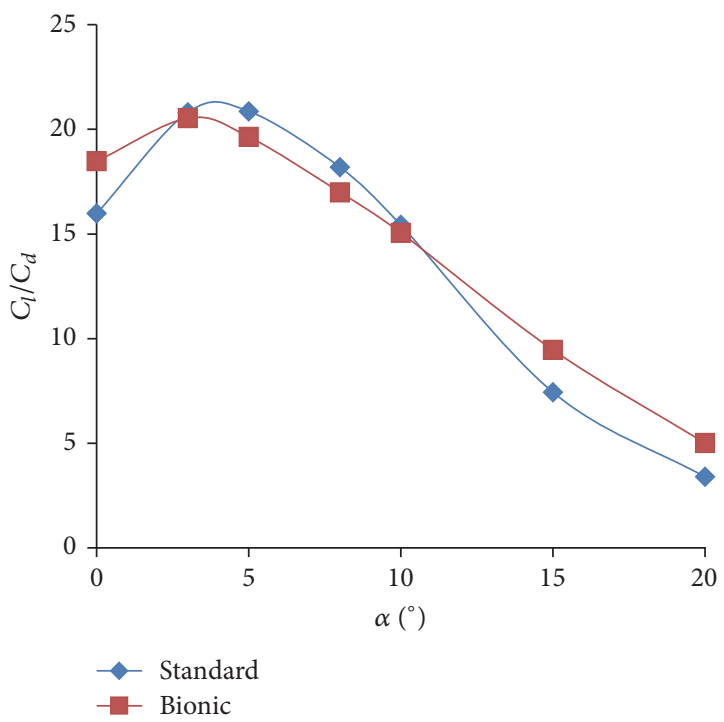

(b) $\operatorname{Re}=8 \times 10^{4}$

FIGURE 16: Lift-to-drag ratio $\left(C_{l} / C_{d}\right)$ curves of the airfoils at different AOA. "Standard" is the lift-to-drag ratio of the standard airfoil; "Bionic" is the lift-to-drag ratio of the bionic airfoil.

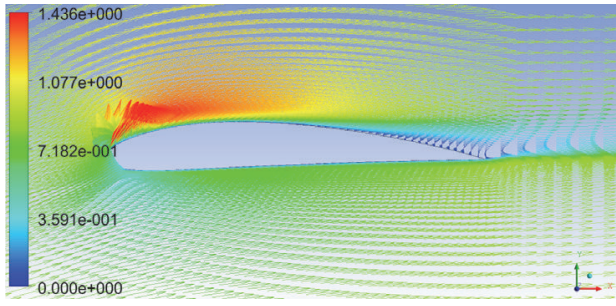

(a) Standard airfoil at $\mathrm{AOA}=10^{\circ}$

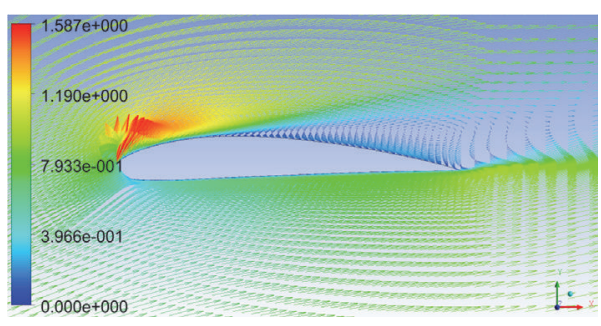

(c) Standard airfoil at $\mathrm{AOA}=15^{\circ}$

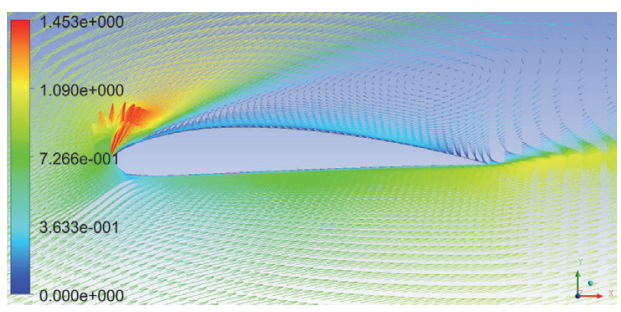

(e) Standard airfoil at $\mathrm{AOA}=20^{\circ}$

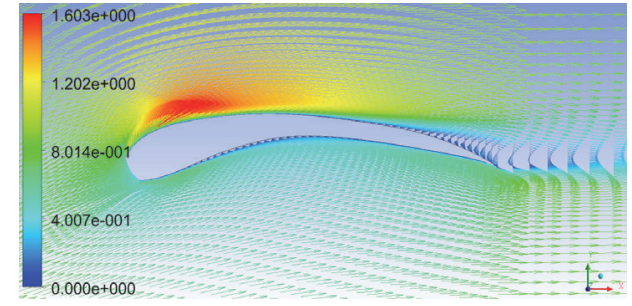

(b) Bionic airfoil at $\mathrm{AOA}=10^{\circ}$

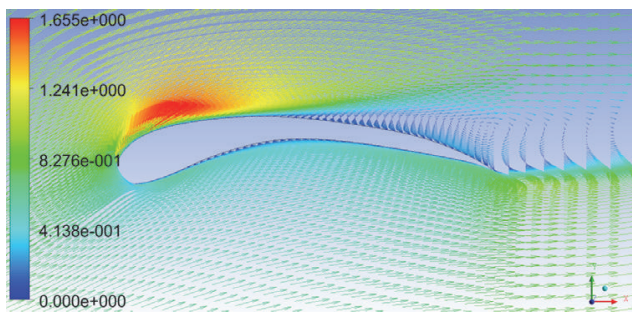

(d) Bionic airfoil at $\mathrm{AOA}=15^{\circ}$

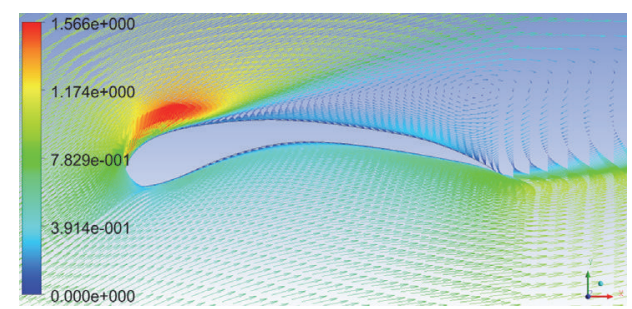

(f) Bionic airfoil at $\mathrm{AOA}=20^{\circ}$

FIGURE 17: The velocity vectors colored by velocity magnitudes at $\mathrm{Re}=6 \times 10^{4}$ at different angles of attack. 


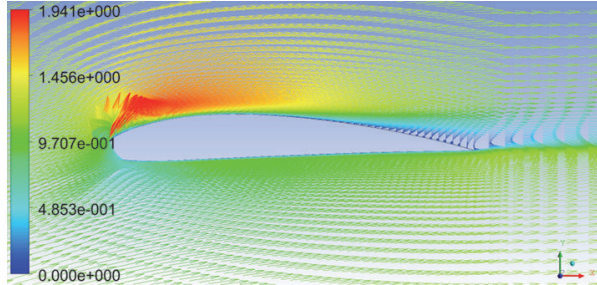

(a) Standard airfoil at $\mathrm{AOA}=10^{\circ}$

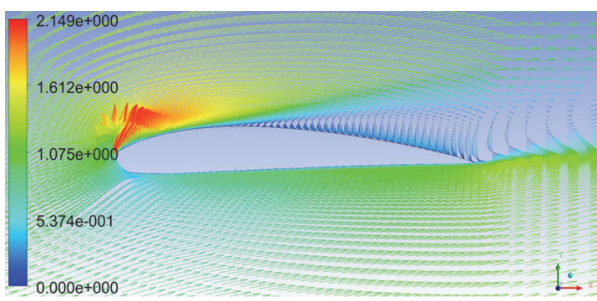

(c) Standard airfoil at $\mathrm{AOA}=15^{\circ}$

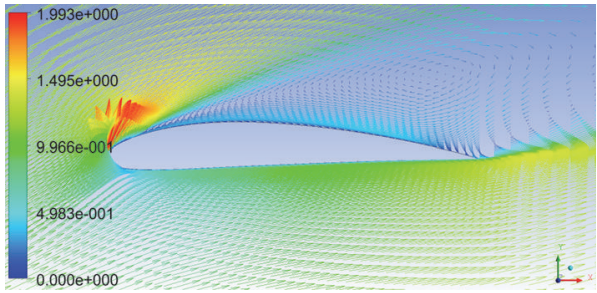

(e) Standard airfoil at $\mathrm{AOA}=20^{\circ}$

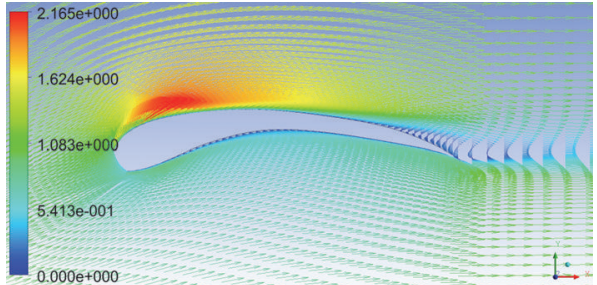

(b) Bionic airfoil at $\mathrm{AOA}=10^{\circ}$

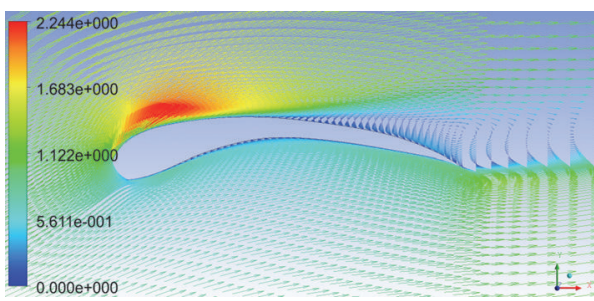

(d) Bionic airfoil at $\mathrm{AOA}=15^{\circ}$

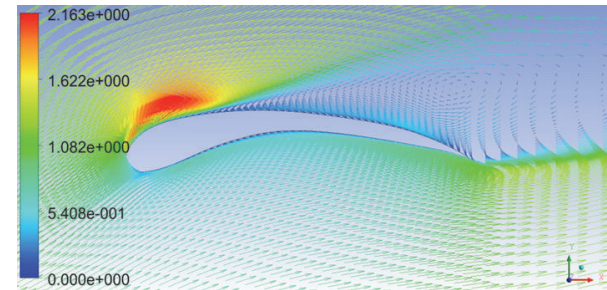

(f) Bionic airfoil at $\mathrm{AOA}=20^{\circ}$

FIGURE 18: The velocity vectors colored by velocity magnitudes at $\mathrm{Re}=8 \times 10^{4}$ at different angles of attack.

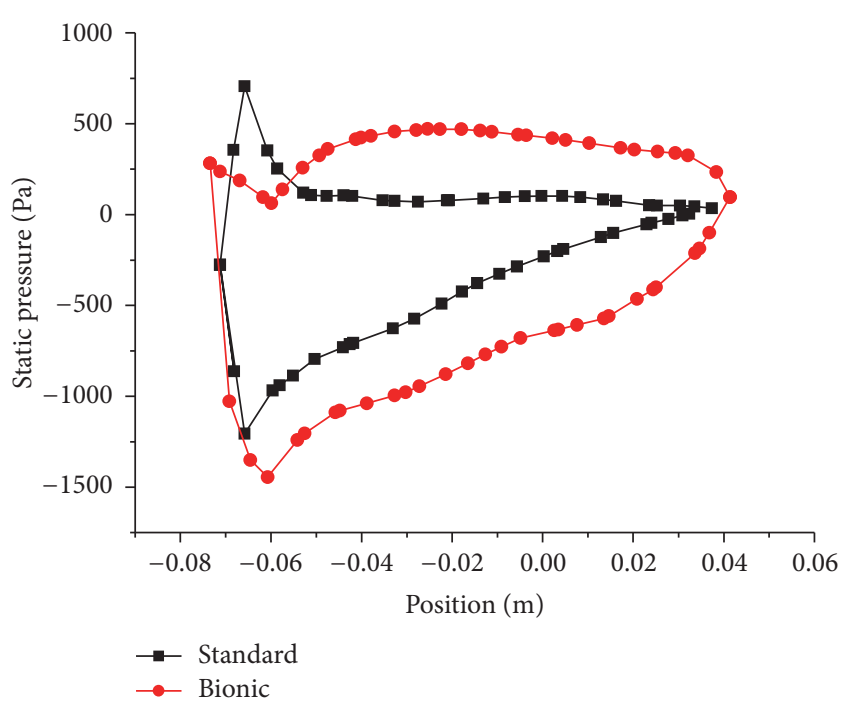

FIGURE 19: Static pressures on windward surfaces of the blades.

standard blade. Such bigger pressure differences can provide larger lift and thus improve the efficiency of the blade. So the application of the bionic airfoil into the blade design is beneficial for the improvement of the efficiency of the blade.

The contours of static pressures of the standard blade and the bionic blade under the wind speed of $9 \mathrm{~m} / \mathrm{s}$ are shown in Figure 20. The pressure at the tip of the standard blade is $398 \mathrm{~Pa}$ and the one at the tip of the bionic blade is $327 \mathrm{~Pa}$, which is noticeably lower. The lower pressure at the tip of the bionic blade decreases the revolving resistance of the blade and improves the efficiency of the blade.

\section{Conclusion}

A bionic method for designing the airfoil was developed under the inspiration of the Long-eared Owl's wings in order to design and process wind turbine blades. Numerical simulation analyses were carried out to compare the difference between the standard airfoil and the bionic airfoil as well as the difference between the standard blade and the bionic blade. In particular, under typical wind speeds and natural conditions, wind turbine efficiency experiments were carried out on the bionic blade and the standard blade.

Compared with the standard airfoil, the bionic airfoil has an increased effective bending as well as an increased effective AOA. The difference in terms of flow velocity between the upper arc and the lower arc is increased. This in turn generates a large pressure difference between the upper and lower surface, which improves the lift of the airfoil. The numerical simulation data show that the stall characteristics of the bionic airfoil are greatly improved compared with the standard airfoil. The superior aerodynamic characteristics of the bionic airfoil play important role on improving the blade 


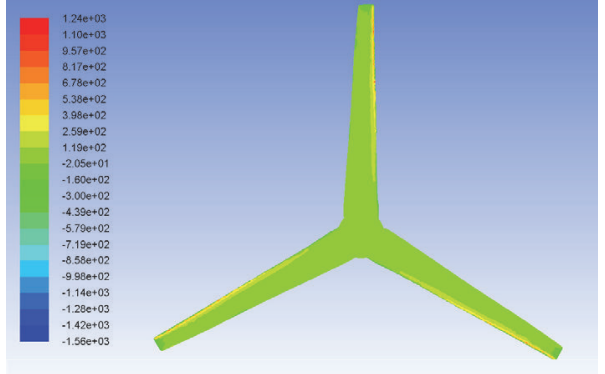

(a) Standard blade

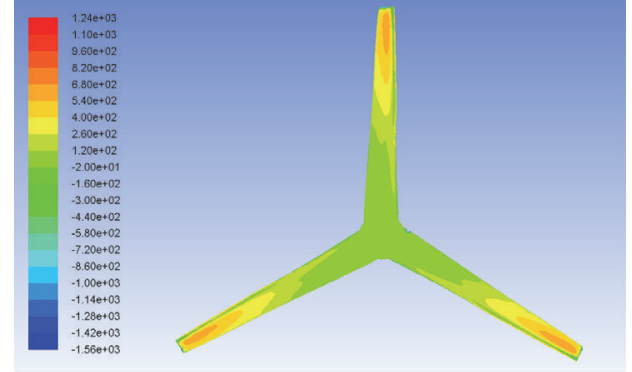

(b) Bionic blade

Figure 20: Contours of static pressures on windward surfaces of the blades.

characteristics. In addition, the bionic blade design inspired by the Long-eared Owl's airfoil has a considerably higher pressure difference between the upper and lower surfaces at the tip than that of the standard blade. Such a larger pressure difference can provide larger lift and thus improve the efficiency of the blade. These results are consistent with those from the wind turbine efficiency experiment.

The bionic design of the blade provides a new method for designing wind turbine blade and both the numerical simulation and the real experimental results will stimulate our interest to further research this topic.

\section{Abbreviations}

AOA: Angle of attack

$C_{p}: \quad$ Power coefficient

$C_{l}: \quad$ Lift coefficients

$C_{d}: \quad$ Drag coefficients

$C_{l} / C_{d}$ : Lift-to-drag ratio.

\section{Competing Interests}

The authors declare that there are no competing interests regarding the publication of this paper.

\section{Acknowledgments}

The authors gratefully acknowledge the support of Scientific and Technological Developing Program of Jilin Province (Grant no. 20140204082GX) and National Science Foundation of China (Grant no. 51305157).

\section{References}

[1] V. Fthenakis and H. C. Kim, "Land use and electricity generation: a life-cycle analysis," Renewable and Sustainable Energy Reviews, vol. 13, no. 6-7, pp. 1465-1474, 2009.

[2] P. Fuglsang and C. Bak, "Development of the Risø wind turbine airfoils," Wind Energy, vol. 7, no. 2, pp. 145-162, 2004.

[3] A. Bjork, Coordinates and Calculations for the FFA-WI-xxx, FFA-W2-xxx and FFA-w3-xxx Series of Airfoils for Horizontal Axis Wind Turbines, FFA TN, Stockholm, Sweden, 1990.
[4] W. A. Timmer and R. P. J. O. M. van Rooij, "Summary of the Delft University wind turbine dedicated airfoils," Journal of Solar Energy Engineering, vol. 125, no. 4, pp. 488-496, 2003.

[5] M. S. Selig and B. D. McGranahan, "Wind tunnel aerodynamic tests of six airfoils for use on small wind turbines," Journal of Solar Energy Engineering, Transactions of the ASME, vol. 126, no. 4, pp. 986-1001, 2004.

[6] J.-Y. Li, R. Li, Y. Gao, and J. Huang, "Aerodynamic optimization of wind turbine airfoils using response surface techniques," Proceedings of the Institution of Mechanical Engineers, Part A: Journal of Power and Energy, vol. 224, no. 6, pp. 827-838, 2010.

[7] A. Hedenström, "Aerodynamics, evolution and ecology of avian flight," Trends in Ecology and Evolution, vol. 17, no. 9, pp. 415422, 2002.

[8] G. R. Spedding, “The aerodynamics of flight," Advances in Comparative and Environmental Physiology, vol. 11, pp. 51-111, 1992.

[9] A. Chehouri, R. Younes, A. Ilinca, and J. Perron, "Review of performance optimization techniques applied to wind turbines," Applied Energy, vol. 142, pp. 361-388, 2015.

[10] Q. Cong, Y.-R. Liu, Y. Ma, and J.-F. Jin, "Aerodynamics analysis of the airfoil when wings of swallow is gliding," Journal of Jilin University (Engineering and Technology Edition), vol. 41, no. 2, pp. 231-235, 2011.

[11] J. Jin, Y. Ma, Y. Liu et al., "Aerodynamic analysis of the family of airfoil of owl," Journal of Jilin University (Engineering and Technology Edition), vol. 40, no. 1, pp. 278-281, 2010.

[12] C.-Y. Xu, Z.-H. Qian, Q.-P. Liu, S.-M. Sun, and L.-Q. Ren, "Aerodynamic performance of bionic coupled foils based on leading edge of long-eared owl wing," Journal of Jilin University (Engineering and Technology Edition), vol. 40, no. 1, pp. 108-112, 2010.

[13] X. Hua, R. Gu, J. Jin et al., "Numerical simulation and aerodynamic performance comparison between seagull aerofoil and NACA 4412 aerofoil under Low-Reynolds," Advances in Natural Science, vol. 3, no. 2, pp. 244-250, 2010.

[14] K. J. Standish and C. P. Van Dam, "Aerodynamic analysis of blunt trailing edge airfoils," Journal of Solar Energy Engineering, Transactions of the ASME, vol. 125, no. 4, pp. 479-487, 2003.

[15] J. P. Baker, E. A. Mayda, and C. P. van Dam, "Experimental analysis of thick blunt trailing-edge wind turbine airfoils," Journal of Solar Energy Engineering, vol. 128, no. 4, pp. 422-431, 2006. 


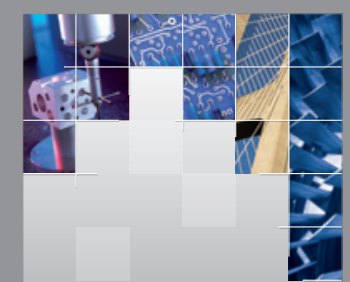

\section{Enfincering}
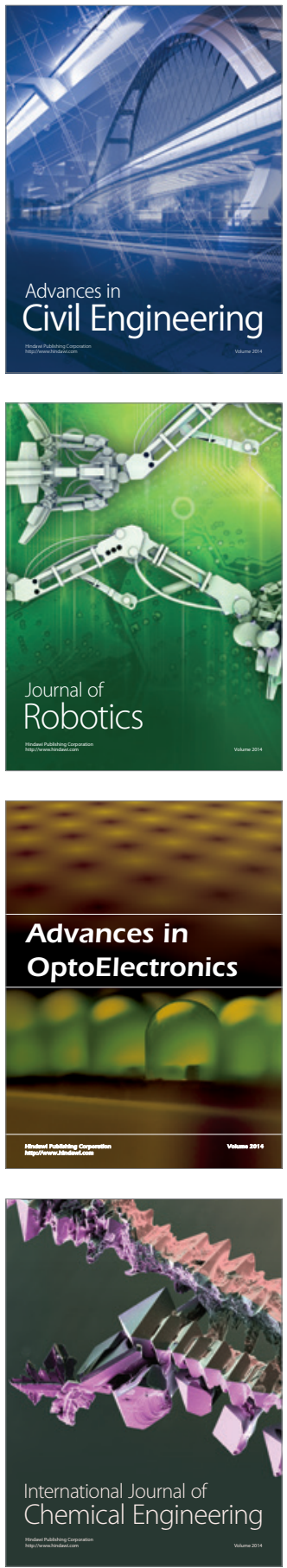

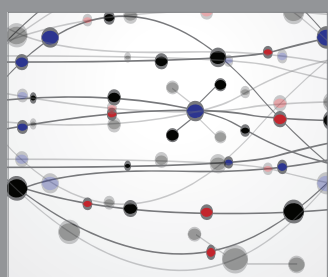

The Scientific World Journal

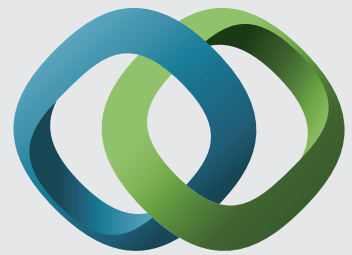

\section{Hindawi}

Submit your manuscripts at

https://www.hindawi.com
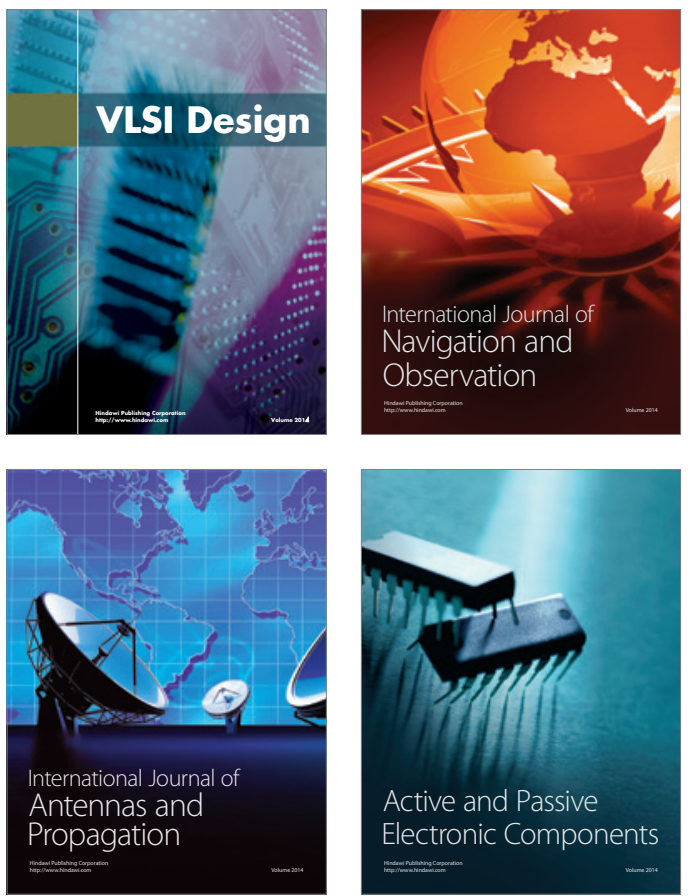
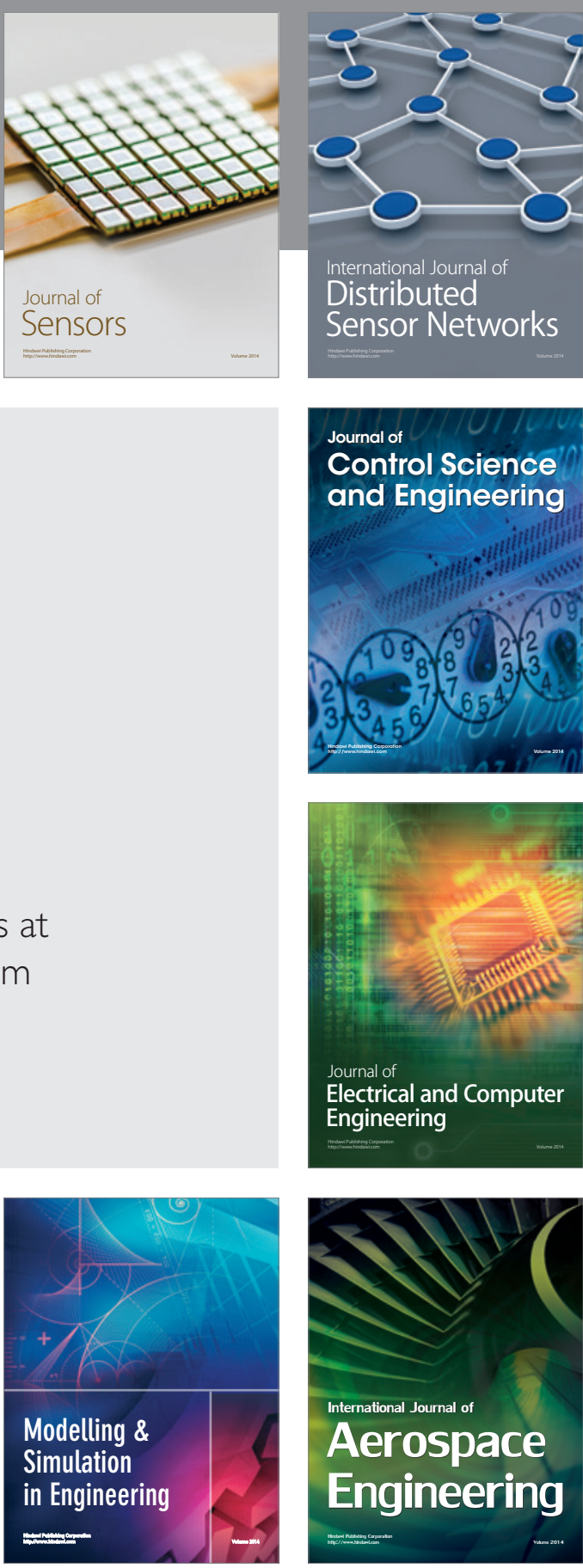

International Journal of

Distributed

Sensor Networks

$-$

Joumal of

Control Science

and Engineering
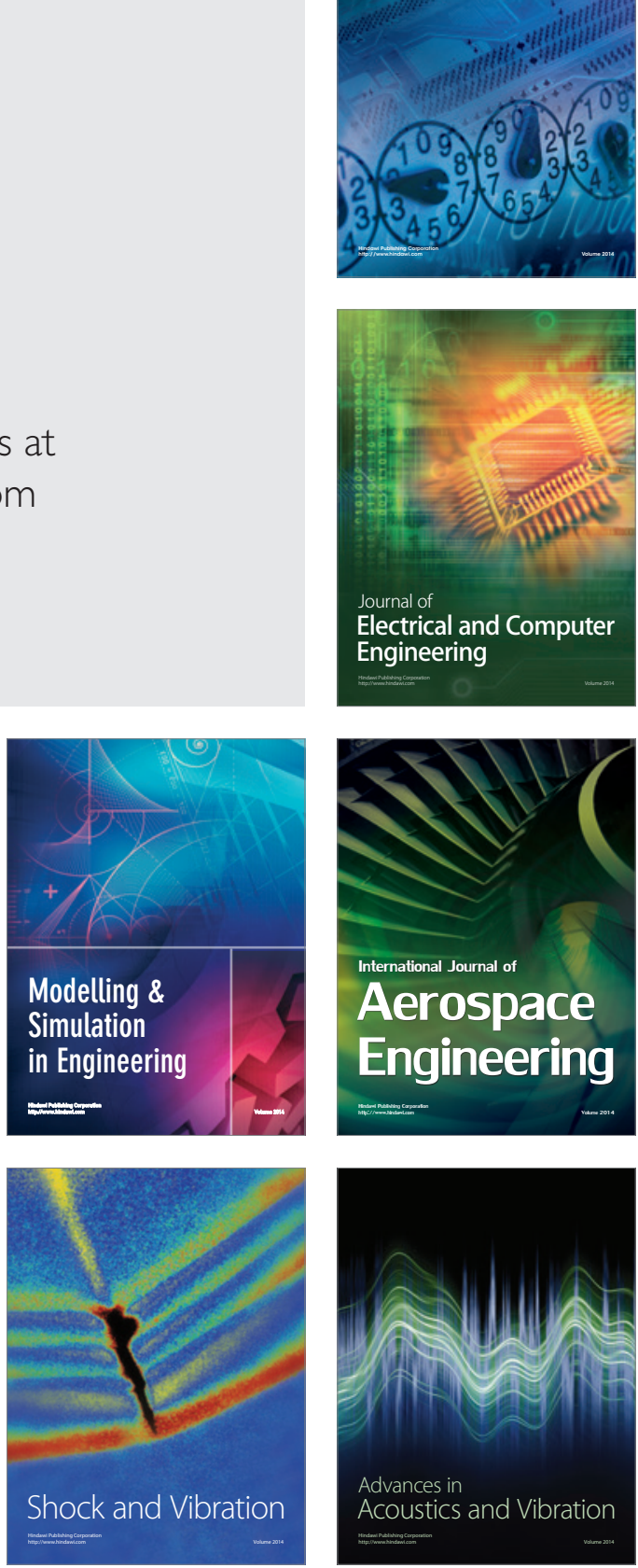